\title{
Androcentrismo, ciencia y filosofía de la ciencia
}

\author{
Androcentrism, science and philosophy of science
}

\author{
Federico Nahuel Bernabé \\ Universidad Nacional Arturo Jauretche; \\ CEFHIC-Universidad Nacional de Quilmes, Argentina \\ fnbernabeblach@gmail.com
}

\begin{abstract}
Resumen
En este trabajo retomaremos los aportes de la filosofía feminista de la ciencia en torno al androcentrismo, con especial énfasis en la biología y las ciencias biomédicas. Propondremos que tales aportes pueden ordenarse atendiendo a tres sentidos distintos de androcentrismo, y que entre tales sentidos aparecen tensiones importantes. Siguiendo el camino trazado por Longino, el empirismo crítico contextual, defenderemos que la reconstrucción racional de teorías puede ayudarnos a precisar dónde se agazapan los vectores de decisión patriarcales en la práctica científica. Para ello, presentaremos un análisis alternativo al de Longino y lo utilizaremos como insumo para discutir la idea de cerebros tipo en el marco de la neuroendocrinología del comportamiento.
\end{abstract}

Palabras clave: androcentrismo, biología, feminismo, estructuralismo, filosofía.

\begin{abstract}
In this work we will take up again the contributions of the feminist philosophy of science around androcentrism, with special emphasis on biology and biomedical sciences. We will propose that such contributions can be ordered according to three different senses of androcentrism, and that important tensions appear between these senses. Following the path traced by Longino, contextual critical empiricism, we will defend that the rational reconstruction of theories can help us to specify where patriarchal decision vectors crouch in scientific practice. To this end, we will present an alternative analysis to Longino's and use it as an input to discuss the idea of type brains in the framework of the neuroendocrinology of behaviour.
\end{abstract}

Keywords: androcentrism, biology, feminism, structuralist view of theories, philosophy.

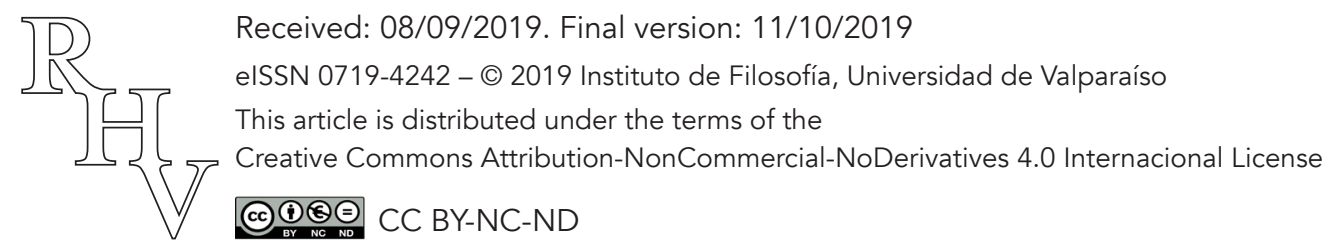




\section{La(s) filosofía(s) feminista(s) de la ciencia y la polisemia del androcentrismo}

Hablar de la filosofía feminista de la ciencia (FFC) como una corriente o escuela de pensamiento bien delimitada constituye una simplificación inaceptable. Consideramos más adecuado hablar de un movimiento filosófico, pues el rótulo FFC se aplica a líneas de pensamiento fuertemente heterogéneas, las cuales comparten a lo sumo una orientación general y algunas ideas centrales. Esa mínima común orientación puede precisarse como sigue:

1. La afirmación de la existencia de valores extracognitivos de naturaleza androcéntrica en ciencia.

2. La afirmación de que tales valores son ineliminables.

\section{Porque,}

3. los y las científicas son sujetos cognoscentes situados (situated knowers).

Ahora bien, la situacionalidad del conocimiento y la ineliminabilidad de los valores no son, por supuesto, aportes originales de la FFC, más bien es un lugar común en la filosofía de la ciencia postkuhneana (Gómez 2015). El primer rasgo propio y característico de la FFC es la atención al problema del androcentrismo, dentro del problema general de los valores. Además, los trabajos de la FFC y de la crítica feminista de la ciencia en general constituye el acervo de ejemplares más grande del modo en el que los valores (en este caso, androcéntricos) performan la actividad y los productos científicos. El segundo rasgo, es un fuerte compromiso militante con la transformación social y por ende con la transformación de la ciencia.

Hasta aquí las similitudes qua movimiento filosófico. Cómo presentar las distintas corrientes, sus divergencias y solapamientos, constituye en sí mismo un problema. Si bien la primera taxonomía propuesta por Sandra Harding (1986) que distingue entre empirismo, perspectivismo (standpoint theory) y posmodernismo, ha sido la más difundida, nos inclinaremos por la propuesta más reciente de Elizabeth Potter (2006). En concreto, sirve a nuestros fines pues propone ordenar las corrientes atendiendo no cuestiones de marco sino al modo en el que responden al desafío de la ciencia cargada de valores (patriarcales): de más normativas a más naturalistas.

Representado por Harding $(1986 ; 1993)$ en su versión clásica o estándar y por Alison Wylie (2003) en una versión revisada y más cercana al empirismo, el perspectivismo feminista hereda de la epistemología marxista el rechazo del sujeto ahistórico de conocimiento y la pretendida neutralidad respecto al punto de vista desde el que se construye el conocimiento. Así mismo, sostiene que la perspectiva del grupo social oprimido (las mujeres, pero no sólo, también la raza, la clase social etc.) aporta lo que se ha dado en llamar objetividad fuerte, frente a una objetividad débil de la perspectiva del opresor/dominante. Y esto, porque el oprimido ve todo lo que ve el opresor y además todo aquello que a aquél

Revista de Humanidades de Valparaíso, 2019, No 14, 287-313 
se le escapa. En este sentido, la standpoint theory defiende una epistemología sostenida desde los estilos cognitivos del grupo oprimido, que no sólo suponga un empoderamiento de este sino una mejora epistémica para el conjunto. Así, el perspectivismo es una teoría crítica al estilo de la escuela de Frankfurt, con un fortísimo componente pragmático y emancipatorio.

Empirismo contextual: fundamentalmente sostenido por Helen Longino (1994; 1997) es una de las posturas más fructíferas del feminismo en filosofía de la ciencia. Lo que Longino defiende es que la buena lógica y la evidencia empírica son necesarias pero no suficientes para dar cuenta de la justificación y el rechazo-aceptación de teorías. La actividad científica conlleva esencialmente supuestos de contorno, elementos volicionales sociales y políticos que juegan el rol de vectores de decisión. Hasta aquí, la propuesta es más o menos estándar a partir de la obra de Kuhn, pero lo profundamente original es la propuesta de objetividad de la autora: dado que siempre hay valores, la objetividad se construye a partir de la elucidación de aquellos valores operantes en las distintas teorías/ hipótesis y la discusión crítica intersubjetiva de los mismos. Esto permite garantizar el control de las teorías no sólo en el nivel fáctico o conceptual, sino también axiológico.

Empirismo feminista naturalizado: heredera directo de Quine, la propuesta de Lynn Hankinson Nelson $(1990 ; 1993)$ consiste en tomarse en serio el holismo empirista quineano: no hay diferencias fuertes entre observación y teoría, la filosofía y la ciencia son disciplinas cuya tarea es continua. La filosofía debe brindar una teoría acerca del modo en el cual se construyen teorías científicas mejores. La normatividad aquí es nula: por el holismo contrastacional sabemos que cuando una teoría es puesta a prueba, lo hace el sistema conceptual completo en el que ocurre, incluidas creencias de sentido común y valores epistémicos y no epistémicos. Dado que no hay una distinción interesante entre hechos y valores, no podemos decir que la comunidad de científicos que sostiene posiciones patriarcales hace mala ciencia. En términos de la acción filosófica, parece llamar a una especie de quietismo naturalista.

Dentro de esta riqueza de posiciones, en este trabajo nos ubicaremos en la órbita del empirismo contextual crítico de Longino. Las razones para ello son múltiples y no se presentarán en detalle, pero vale señalar nuestro argumento central: tiene la dosis justa de normativismo como para que el trabajo de análisis filosófico tenga sentido. Como ya señalamos, y por muy sugestivo que resulten las posiciones naturalizadas, subsiste el problema del quietismo filosófico, depositando sobre la sociedad y sus procesos la desaparición de los valores patriarcales de la labor científica. En la otra esquina, el perspectivismo dinamita toda idea de corrección parcial de la ciencia, tanto en su versión marxista como posmoderna: todo es ideología, por lo que el trabajo analítico y crítico de la filosofía de la ciencia sería estéril. Frente a estos tigris y éufrates, se extiende el camino de Longino, cuya posición metodológica parte de que "the logical and cognitive structures of scientific inquiry requires such interaction" (Longino 1990, 5.) No es que los valores no epistémicos como vectores de decisión sean contrabando indeseable en la actividad científica, 
sino que dada la infradeterminación de la teoría por los hechos, tales vectores de decisión valorativos son esenciales a la actividad científica. Justamente, lo interesante de la propuesta longiana es la aceptación positiva de los valores no epistémicos y la búsqueda de una salida al problema de la objetividad que no suponga en algún sentido la negación de estos. Pues es precisamente en la negación, la invisibilización de los valores no epistémicos donde reside la reproducción científica de la opresión.

La solución pasa entonces por la discusión racional de los valores presupuestos por las teorías en disputa. Para ello, se establecen cuatro condiciones básicas o normas para el empirismo contextual crítico (Borgerson 2011):

1. Espacios reconocidos para el criticismo, es decir, deben existir revistas académicas, congresos u otras instancias reconocidas por la comunidad científica en las que se generen discusiones críticas acerca del desarrollo de una disciplina dada.

2. El criticismo no debe ser ignorado, esto es, los y las científicas deben hacerse cargo de las revisiones críticas de sus pares.

3. Estándares públicos y compartidos. La crítica efectuada por los pares requiere la aceptación compartida de ciertas reglas que han de ser públicas para garantizar que todos y todas las participantes de la discusión hablan de lo mismo.

4. Razonable igualdad de autoridad, es decir, la aceptación o rechazo de teorías o hipótesis debe residir en la discusión intersubjetiva y no en la autoridad de quién propone tal o cual teoría.

Central al empirismo contextual crítico es, por tanto, la negación del científico individual y ahistórico como sujeto de la ciencia. En una clara herencia historicista, Longino revaloriza la práctica colectiva de la ciencia como instancia de decisión racional de los valores no epistémicos presupuestos en la práctica científica. Finalmente, lo más importante para nuestros intereses es que es un presupuesto para la práctica del FFC que los valores deben ser explicitados. Ahí es donde entendemos que la filosofía qua análisis elucidatorio y reconstructivo puede jugar un rol importante, tal y como la misma Longino dice y muestra.

\subsection{Los tres sentidos de androcentrismo}

Aun cuando aquí seguiremos el camino programático de Longino, la denuncia de la persistencia de sesgos androcéntricos o valores patriarcales en la práctica y en los productos científicos es un resultado general de la FFC en su conjunto. Ahora bien, defenderemos que androcentrismo se dice de al menos tres maneras, las cuales están estrechamente vinculadas, pero son distinguibles y en ciertos casos pueden generar tensiones internas. 
En primer lugar, androcentrismo se dice en un sentido sociológico y se evidencia en la escasez de mujeres y cuerpos feminizados en la ciencia. Esta escasez no es ni única ni principalmente numérica, sino respecto a los cargos y puestos de poder dentro de las comunidades. Así mismo, el sesgo androcéntrico sociológico se manifiesta en la feminización de ciertas áreas en detrimento de otras, el reparto de tareas en los laboratorios y en el trabajo de campo. Esta dimensión ha sido ampliamente explorada en los países centrales y se vinculó con los primeros pasos de la crítica feminista de la ciencia, e incluso con las primeras políticas inspiradas en estas críticas (incorporación de las mujeres a la ciencia, la idea de mujer científica). Para una revisión de literatura extensa y reciente, puede consultase Tacsir et al. (2014).

El segundo sentido de androcentrismo apunta a la línea de flotación de la objetividad científica, el androcentrismo conceptual/explicativo: la elección de teorías está sesgado en favor de aquellas que reproducen y naturalizan el estatus quo opresivo hacia las mujeres. Las comunidades científicas favorecen aquellas teorías que van mano a mano con el prejuicio sobre la inferioridad intelectual de las mujeres.

Este androcentrismo explicativo/conceptual ha resultado protagónico en los trabajos más importantes de la FFC, particularmente en áreas como la biología evolutiva, la arqueología (Wylie 1997), la neurociencia y las ciencias del comportamiento (Fausto-Sterling 1992). En cada una de esas áreas las autoras han mostrado cómo las teorías que mantenían una perspectiva favorable a la dominación masculina han sido mejor aceptadas que otras, incluso, han sobrevivido a abundante evidencia contraria. La posición general tomada a partir de estos hallazgos consiste en dudar de toda perspectiva científica que aborde, desde una perspectiva biológica, las diferencias entre machos y hembras de nuestra especie. Esta duda o sospecha va desde una lectura crítica de los resultados obtenidos por tales perspectivas biológicas, hasta la calificación de mera ideología de estos trabajos.

Finalmente, hay un tercer sentido de sesgo androcéntrico al que hemos dado en llamar subrogativo y consiste en la exclusión de las mujeres y la diversidad qua objetos de estudio de las ciencias. Particularmente potente en el área biológica y biomédica, el sesgo surrogativo toma como modelo para la producción del conocimiento al hombre. Lo más interesante es que este sesgo se extiende más allá de nuestra especie, y ocurre incluso en el trabajo experimental con animales (Bernabé y Giri 2019). Dicho de una vez, el macho de las especies es generalmente tomado como ejemplar de la especie, excepto cuando se estudia la reproducción. Aun cuando ha sido menos potente dentro de la FFC es una de las formas en que la perspectiva de género ha generado un fuerte efecto en el mainstream, apareciendo toda una nueva disciplina de gendered medicine (Wizemann y Pardue 2001; Legato 2004; Klinge y Wiesemann 2010; Regitz-Zagrosek 2012).

Mientras que la posición general tomada a partir del androcentrismo conceptual/explicativo es la sospecha respecto al estudio de las diferencias sexuales entre mujeres y 
hombres, la posición general tomada a partir del androcentrismo subrogativo parece ir en una dirección contraria, pues la solución a la medicina que toma como objeto modélico al hombre pasa por el estudio de las diferencias y la diferenciación sexuales.

\section{Neurosexismo, la forma contemporánea del androcentrismo explicativo}

En este apartado vamos a profundizar en el segundo sentido de androcentrismo apenas presentado, que en su versión más contemporánea toma el nombre de neurosexismo. El neologismo fue acuñado por la filósofa de la ciencia Cordelia Fine en su comunicación breve de 2008: "Will Working Mothers' Brains Explode? The Popular New Genre of Neurosexism" y posteriormente desarrollado en profundidad en su libro "Delusion of Gender" (Fine 2010). Si bien tomaremos como punto de partida para caracterizar la noción el trabajo de síntesis de Fine, debe notarse que el término por ella acuñado nombra un concepto que es resultado del trabajo mancomunado de distintas filósofas y científicas feministas.

\section{1 ¿De qué hablamos cuando hablamos de neurosexismo?}

En la obra de Fine $(2010 ; 2008)$, "neurosexismo" tiene al menos tres significados notablemente distintos, si bien no son correctamente diferenciados sino más bien presentados de forma ecléctica a través de ejemplos procedentes tanto de literatura científica como de publicaciones divulgativas. Veamos algunos ejemplos:

Como el mago que sabe que vas a elegir el siete de diamantes antes de incluso abrir el mazo, Sarah puede sorprender a su esposo a voluntad, gracias a su afortunada habilidad de saber lo que sientes antes incluso de que lo sienta. [...] Sarah no es ni una vidente de parque de atracciones ni la irresponsable dueña de una futurista máquina de interpretación de ondas cerebrales. Ella es simplemente una mujer que disfruta el milagroso don de leer la mente, que aparentemente les pertenece a todas las propietarias de un cerebro femenino [...] Sarah es solo uno de los muchos y curiosos personajes que pueblan los libros de divulgación científica acerca del género. Ella se encuentra en el libro El cerebro femenino de Louann Brizendine (2007), uno de los recientes e influyentes libros defendiendo la existencia de diferencias fundamentales en la psicología de hombres y mujeres, inscriptas en el hardware neural de estos. Desafortunadamente, la precisión científica y el sentido común son a menudo víctimas de la fealdad de acercarse al anticuado sexismo, formulado en el respetable y autoritario lenguaje de la neurociencia. (Fine 2008, 69)

Es decir, aquí neurosexismo se aplica al género de divulgación cientifica que utiliza nociones de la neuroendocrinología del comportamiento y de las neurociencias en general para establecer las diferencias entre el cerebro de las mujeres y los hombres, las cuales explican a su vez el instinto emocional femenino. Además, este género responde a la an-

Revista de Humanidades de Valparaíso, 2019, No 14, 287-313 
ticuada posición ideológica del sexismo. Ahora bien, Fine no sólo carga las tintas contra esta divulgación científica ideologizada, sino que además apunta directamente a la teoría organizacional Activacional (OR):

Tales descubrimientos condujeron a una idea brillantemente elegante conocida como la hipótesis organizacional-activacional [...] Y así, los neuroendocrinólogos han investigado la intrigante idea de que la testosterona prenatal organiza el cerebro. Manipulan los entornos hormonales de los animales durante el período crítico en el que se cree que tiene lugar la organización del cerebro, y ven qué les sucede a sus cerebros y su comportamiento. [...] ¿Podría la testosterona prenatal en los seres humanos crear diferencias sexuales permanentes en el cerebro que se encuentran detrás de las diferencias de género en la cognición y el comportamiento? [...] Los investigadores hipotetizan que los cambios que ven en el comportamiento son resultado de sus manipulaciones hormonales, es decir, que se producen por cambios inducidos por la testosterona en el cerebro del feto (o, en el caso de la rata, el cerebro neonatal). Y digo hipotetizan porque ha resultado más difícil de lo que se podría pensar $[\ldots]$ conectar los puntos entre las hormonas prenatales y los cambios cerebrales. (Fine 2010, 119)

En el tratamiento de la OR que hace Fine se presentan de modo notablemente simplificado los presupuestos de la teoría e inmediatamente se introduce la sospecha de cuán acertada sea esta teoría, particularmente en el caso de los humanos. La posición de Fine parece apuntar a una visión relativamente compartida con otras autoras críticas de la OR que podemos sintetizar como sigue: la teoría funcionaría razonablemente bien para algunas aves como los pinzones cebra y en mamíferos no humanos. En humanos, en cambio, la conexión causal entre hormonas, estructuras neurofisiológicas y conductas se pierde. Pero no es sólo eso lo que se señala respecto del estatus de la OR. Además de ser dudosa la aplicación del marco conceptual en el caso de los mamíferos de mayor volumen cerebral, algunos pasajes de la historia reciente de la disciplina parecen indicar que la neuroendocrinología tiende a sostener teorías incluso contra la evidencia fáctica Como ejemplo cita a Geschwind:

Norman Geschwind y sus colegas sugirieron una teoría muy compleja, que involucraba la idea de que el alto nivel de testosterona fetal experimentado por los hombres ralentiza el crecimiento del hemisferio izquierdo del cerebro. Geschwind llegó a sugerir que esto deja a los hombres con un mayor potencial para 'talentos superiores en el hemisferio derecho, como el talento artístico, musical o matemático’. (Fine 2010, 122)

Debe notarse aquí en primer lugar, que la posición de Greschwind no se corresponde con los casos paradigmáticos tratados en nuestra reconstrucción. La teoría bajo análisis trata de estructuras dimórficas discretas como la SDN-POA o el HV vinculadas con conductas bien delimitadas. El autor discutido por Fine, en cambio, apunta a un dimorfismo 
cerebral global que se corresponde con un dimorfismo cognitivo general. En segundo lugar, a lo que apunta la autora no es a que Greschwind o la OR sean o no la misma teoría, sino más bien a que ambas comparten cierta inmunidad respecto a las refutaciones:

La teoría de Geschwind es la sartén teflón de la literatura cientifica. Mientras que otras teorías se vuelven sucias e inutilizables cuando se arroja sobre ellas datos desconfirmantes, estos simplemente resbalan sobre teoría de Geschwind, que continúa sobreviviendo e inspirando a pesar de las críticas importantes que apuntan a la conclusión de que el estado actual de la teoría debería ser una ambiciosa-idea-que-no-funciona. (Fine 2010, 122)

El argumento entonces es que, teniendo claros casos en contra, la teoría no es descartada de forma inmediata por parte de la comunidad, y que inspira (asumimos que aquí Fine hace referencia a la heurística que genera) la investigación en el área. A lo que se apunta pues es que "[c]on todo, la idea de que mayores niveles de testosterona fetal de alguna manera crean los cerebros masculinos" que son, claro, superiores en actividades típicamente masculinas. Es decir, independientemente de que Gerschwind y la OR sean la misma teoría, lo que comparten (el recurso a la acción organizacional de las hormonas en períodos críticos del desarrollo fetal para explicar las conductas dimórficas) se sostiene pese a la evidencia en contra. Y esta irracionalidad por parte de la comunidad de endocrinólogos del comportamiento se explica porque se condice bien con ciertos prejuicios acerca de lo que significa ser hombre y ser mujer.

A la luz de lo apenas dicho, no es difícil ver que neurosexismo es polisémico en tanto se predica de distintas cosas:

A) En primer lugar, nombra una categoría literaria de divulgación científica en la cual se utiliza como insumo ciertos desarrollos neurocientíficos y su lenguaje para sostener que la desigualdad entre hombres y mujeres es resultado de sus diferencias biológicas.

B) En segundo lugar, nombra la posición ideológica que sostiene que la desigualdad entre hombres y mujeres es resultado de sus diferencias biológicas, particularmente las diferencias neuroendocrinas.

C) Por último, nombra la propia neuroendocrinología (la OR) en tanto sirve como dudoso sustento científico de A y B.

\footnotetext{
${ }^{1}$ Debe notarse que Fine utiliza la expresión "lay science" cuya traducción literal sería ciencia lega. En general, lay science hace referencia a aquellos artículos periodísticos u otras obras de divulgación escritas por periodistas y/o divulgadores profesionales. No obstante, algunas de las obras discutidas por la propia autora, aun siendo netamente divulgativas, son escritos por miembros de la comunidad científica. Por ello, hemos elegido traducirlo por el término más general de divulgación.
} 
Debe notarse que neurosexismo es auténticamente polisémico, en tanto las caracterizaciones propuestas A-C no son intercambiables en ningún sentido, dado que nombran realidades bien distintas: cierta literatura divulgativa, cierta posición ideológica y cierta área de experticia científica. Si bien la autora pretende enfatizar el modo en el cual estos tres elementos están vinculados, no cabe duda de que pueden ser atacados o defendidos de forma independiente. No es necesario, por ejemplo, aceptar el estatus dudoso de la neuroendocrinología para reconocer la existencia de una posición ideológica sexista, y lo mismo con el género de divulgación científica.

\subsection{La larga marcha de la opresión científicamente justificada}

Aun si tenemos razón en sostener que neurosexismo se dice de cosas distintas que son independientes entre sí, las ideas de Fine estarían solo pobremente presentadas si obviamos uno de sus puntos centrales: el neurosexismo vincula estos tres elementos, reeditando la conocida crítica feminista a la ciencia que la propia autora cita al inicio de la sección pertinente del libro:

Durante dos milenios, los "expertos imparciales" nos han dado ideas tan incisivas como el hecho de que las mujeres carecen de suficiente calor para hervir la sangre y purificar el alma, que sus cabezas son demasiado pequeñas, sus vientres demasiado grandes, sus hormonas demasiado debilitantes, que piensan con sus corazones o con el lado equivocado del cerebro. La lista nunca termina. (Hess 1990, 81)

Lo que Fine sostiene, a final de cuentas, es que la neuroendocrinología del comportamiento es un capítulo más de lo que podemos llamar la larga marcha de la opresión científicamente justificada. Si miramos al menos de forma breve esa historia a la que se refieren Hess, Fine y otras autoras y autores, encontramos convincente evidencia de que hay coincidencias entre las concepciones ideológicas sobre cuáles son las diferencias entre hombres y mujeres, y las explicaciones brindadas al respecto. Ya en la obra sobre la naturaleza de Aristóteles nos encontramos con propuestas explicativas acerca de las diferencias conductuales entre machos y hembras que cifran estas en la constitución biológica de los sujetos. En concreto, de acuerdo con Aristóteles es la capacidad diferencial de cocer la sangre de machos y hembras la que explica el desarrollo inferior de las hembras, asignándole no sólo determinadas funciones reproductivas sino además su carácter natural sumiso, débil y su escasa aptitud intelectual. Dando un súbito salto secular, en los últimos capítulos de El origen del hombre Darwin se ocupa de dejar en claro que las diferencias entre hombres y mujeres, en lo tocante a lo intelectual, se explican por la selección.

La principal distinción en la capacidad intelectual de los dos sexos queda demostrada porque el hombre alcanza una mayor eminencia, en cualquier cosa que emprenda, que la que pueda alcanzar la mujer [...] los machos han de defender a sus hembras, así como a sus jóvenes, de enemigos de todo tipo, y cazar para su subsistencia conjunta. Pero evitar a los

Revista de Humanidades de Valparaíso, 2019, No 14, 287-313 
enemigos o atacarlos con éxito, capturar animales salvajes y construir armas, requiere la ayuda de facultades mentales superiores, a saber, observación, razón, invención o imaginación. Estas diversas facultades se habrían puesto así continuamente a prueba y se habrían seleccionado durante el estado adulto [...] (Darwin 2009, 740-741)

Independientemente de que hoy exista un feminismo darwinista, parece claro que uno de los resultados que Darwin consideraba razonable a partir de sus teorías es la natural inferioridad de la mujer respecto de los hombres. El statu quo opresivo de las mujeres respecto de los machos es resultado de la evolución. Unos años más tarde, en 1915 el reputado neurólogo Dr. Charles L. Dana cargaría las tintas contra el movimiento sufragista, aduciendo que la constitución del sistema nervioso femenino las hace menos aptas para el ejercicio racional de la elección de representantes y la búsqueda del bien común. Es más, votarlas las pondría en un severo riesgo de padecer enfermedades psiquiátricas graves, por lo que el sufragismo atentaba contra la salud pública de esa "preciosidad inestable" (sic.) que es el sexo femenino. Como señala Hess, la lista parece nunca acabar.

Lo que se pone en juego en todos estos ejemplos es lo que Reinharz (1986) denomina low threshold for male dominance theorizing (bajo umbral para la teorización de la dominación masculina). Es decir, los mecanismos de aceptación o rechazo de teorías son sesgados en favor de aquellas teorizaciones que favorecen la posición ideológica de la superioridad masculina. Pensando ahora en términos de neurosexismo, la neuroendocrinología del comportamiento, al ser evaluada a la luz de esta crítica feminista de la ciencia, se muestra como mala ciencia o, como mínimo, dudosa. Con distintos grados de fortaleza, la idea de que la OR ha recibido un trato preferencial debido a que refuerza los prejuicios sexistas y discriminatorios hacia la mujer y otras minorías en términos de derechos, puede hallarse en van der Wijngaard (1997), Brookey (2002) Jordan-Young (2010) y Anne Fausto Sterlling (1992). No obstante, quien primero presenta un análisis concreto acerca de la neuroendocrinología del comportamiento es Helen Longino (1997).

El argumento central de Longino es el siguiente: dados los datos disponibles (al momento de realizar el análisis filosófico) podrían subsumirse en otro esquema conceptual que se condice al menos tan bien como la OR con los datos disponibles. Y si es así, si dos teorías pueden hacerse cargo igualmente bien de los datos disponibles, entonces se evidencia que el apoyo a uno u otro se debe a factores valorativos/ideológicos. Discutiremos en profundidad inmediatamente.

\subsection{La reconstrucción de Longino}

Longino llama modelo hormonal lineal a la OR en tanto se caracteriza por "ofrecer explicaciones en términos de caminos causales irreversibles y unidireccionales [..] desde los niveles hormonales en el útero, pasando por la organización cerebral, hasta llegar a la conducta" (Longino 1997, 76). De acuerdo con la autora, el modelo lineal se sustenta

Revista de Humanidades de Valparaíso, 2019, No 14, 287-313 
sobre cuatro tipos de datos: experimentación animal, fisiológicos, anatómicos y conductuales. Con ellos, los miembros del programa neuroendocrino construyen el siguiente esquema explicativo:

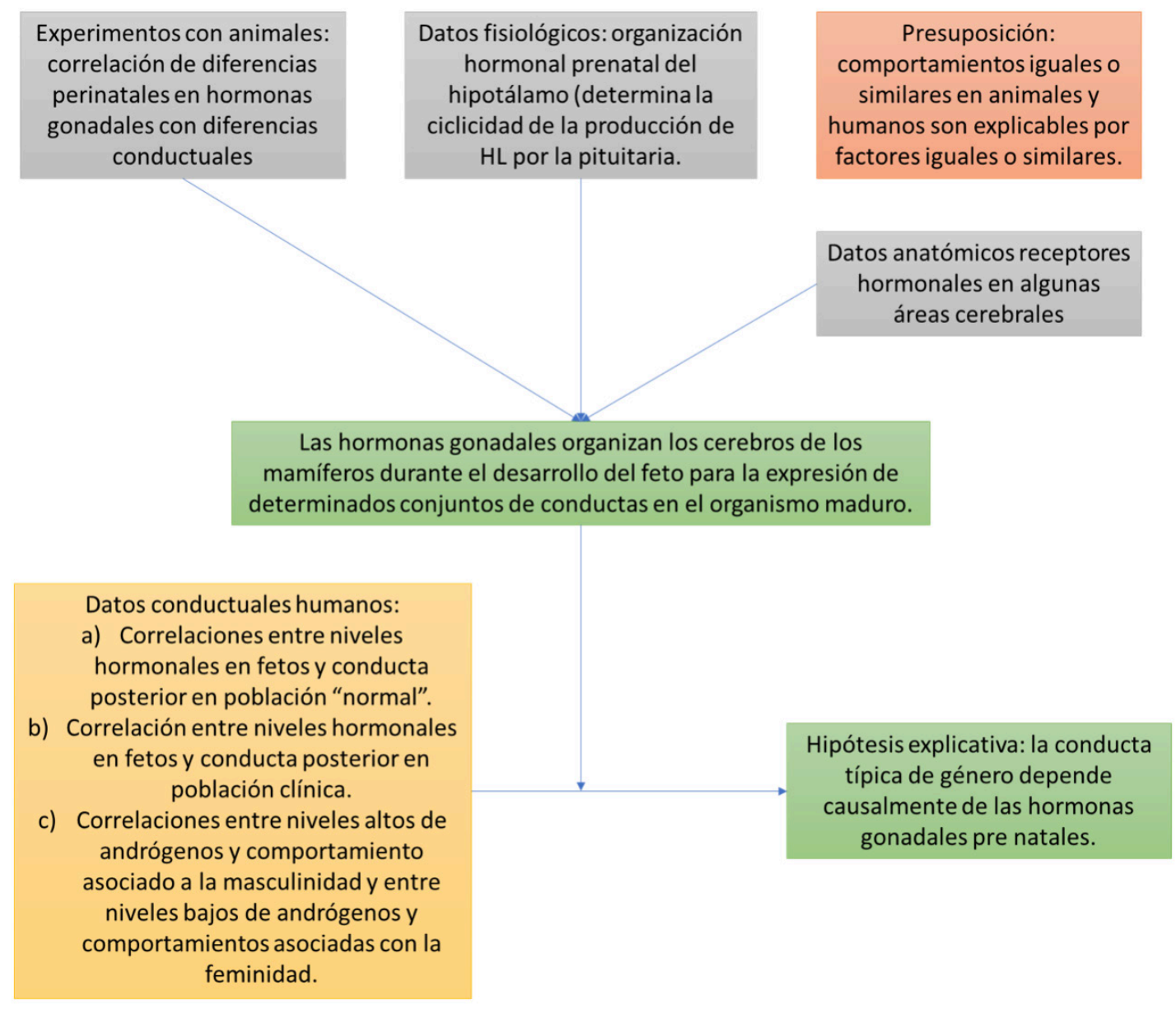

Fig. 1. Elaboración propia a partir del esquema reconstructivo propuesto por Hellen Longino $(1997,77)$.

Nótese que, de acuerdo con la estructura del esquema, los datos provenientes de la fisiología, anatomía y experimentación con animales conducen, junto con la presuposición de que comportamientos iguales o similares en animales y humanos son explicables por los mismos factores, a la afirmación central de la teoría. Posteriormente, dicha afirmación central es aplicada para explicar los datos de los seres humanos, clasificados aquí en función de su procedencia (correlaciones sobre población general y sobre población clínica 
entre hormonas y conductas), generando entonces una afirmación especial y concreta: la conducta típica de género depende causalmente de las hormonas gonadales prenatales que genera dos tipos de cerebros uno masculino y uno femenino.

Ahora bien, si todos estos elementos pudieran ordenarse en otro esquema conceptual, podrían decantarse los supuestos axiológicos subyacentes. Así pues, propone intentar aplicar otro esquema conceptual para ordenar los datos. En este caso, la idea básica es que la interacción de los sujetos humanos con el ambiente social en ciertos períodos especialmente críticos del desarrollo supone una selección (poda o desarrollo) de ciertas conexiones sinápticas, las cuales subyacen a las diferencias cognitivas y conductuales. Este es el nuevo esquema:

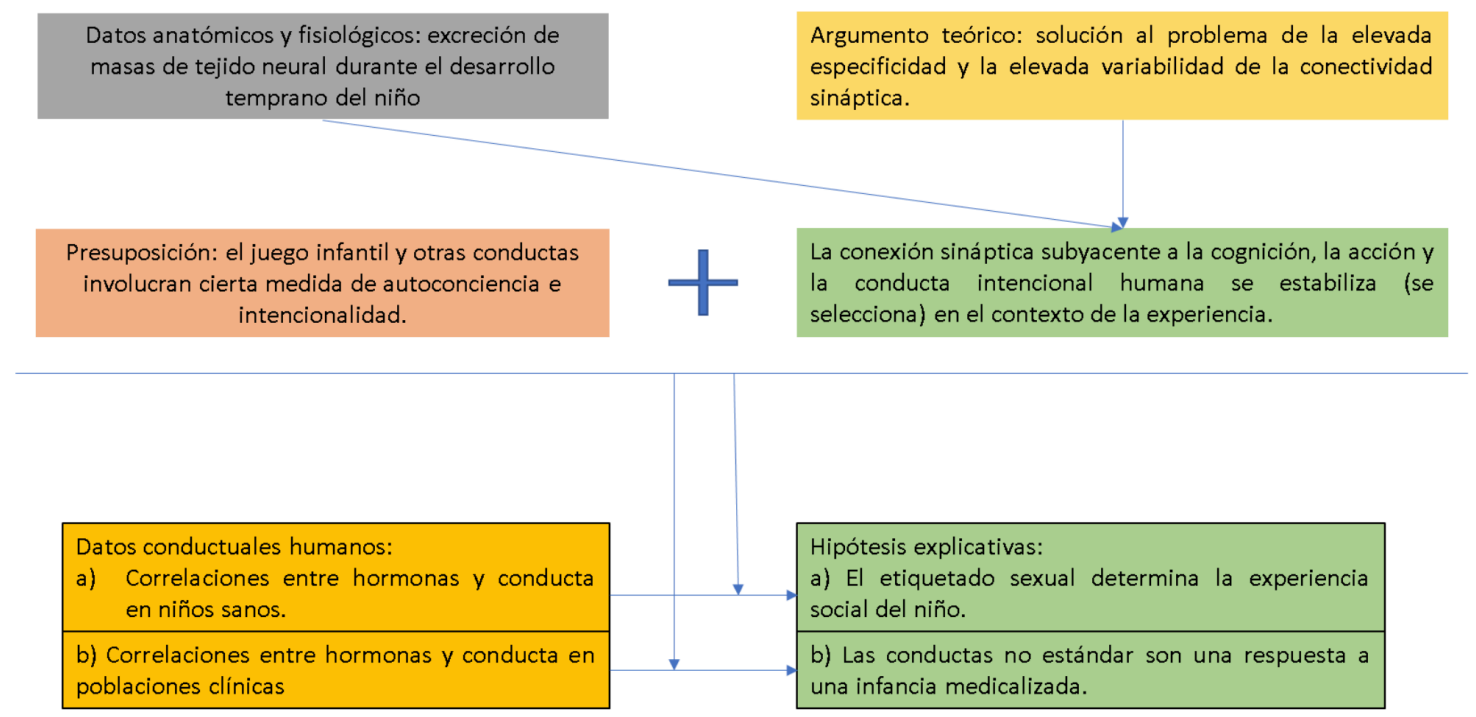

Fig. 2. Elaboración propia a partir del esquema reconstructivo propuesto por Hellen Longino $(1997,78)$.

Ahora bien, los cambios son los esperables a partir de la introducción de una nueva teoría: las aplicaciones específicas a las conductas humanas son distintas pues cifran en el etiquetado social y en la medicalización de la infancia las razones por las que las conductas de hombres y mujeres son distintas. No obstante, aquí hay otros cambios que deben revisarse y que, argumentaré, muestran la necesidad de introducir algunas modificaciones en la noción de empirismo contextual crítico propuesto por Longino. En primer lugar, no es que cambia el "contexto teórico" sino que cambian los datos de partida. En el segundo esquema explicativo se abandona no solo la OR sino también la consideración de los datos procedentes de la experimentación, la fisiología y la anatomía animal. Esto va de la mano con el cambio de presupuesto, en lugar de presuponer la continuidad/extrapolación desde otros mamíferos hacia seres humanos. En segundo lugar, es claro que entre 
el esquema conceptual alternativo propuesto por Longino y la OR no hay coincidencia total en sus aplicaciones pretendidas: mientras que la OR pretende explicar las conductas dimórficas de los mamíferos y al menos algunos vertebrados, la teoría propuesta como alternativa se aplica exclusivamente a los seres humanos.

Como fuere, con estos ingredientes, lo que Longino sostiene es que el éxito de la OR frente a otras alternativas, como la por ella propuesta, se debe a su connivencia con determinados prejuicios profundamente arraigados en la sociedad, al menos en el tratamiento de los seres humanos. No obstante, el análisis presentado por la autora parece mostrar más bien que lo que cambia es el presupuesto de continuidad entre el ser humano y otros mamíferos. Efectivamente, si presuponemos que los seres humanos no pueden ser tratados (en los aspectos relativos a las conductas dimórficas) como el resto de los mamíferos, parece razonable que la OR no se aplique a nuestra especie. Ahora bien, no es en absoluto obvio que adoptar o no tal presupuesto pueda achacarse de modo meridiano a determinados prejuicios ideológicos. Más bien parece un corolario esperable de la biología postdarwin en su conjunto. Si hay un valor ahí, en todo caso es claramente epistémico. Unos parágrafos más adelante, la autora precisa algo más esta idea:

Habiendo visto que los datos pueden recibir distintas interpretaciones en diferentes contex-
tos teóricos, es posible ver el papel de las presuposiciones en la selección y análisis de esos
datos. Los datos sobre comportamiento humano no consisten en correlaciones uniformes,
sino en conjuntos que se agrupan de forma más o menos bipolar. Tomar tal agrupamien-
to como significativo es, en parte, una expresión de la ideología del género, que toma el
género como correlato temperamental y conductual del dimorfismo sexual anatómico y
fisiológico, esto es, una ideología del género que trata el género dimórfico como un rasgo
genuino de la especie. (Longino 1997, 80)

Aquí el argumento cambia y apunta hacia el modo de conceptualizar los datos disponibles sobre la conducta humana. No obstante, este cambio es sólo aparente. De lo que se acusa a los y las científicas bajo la OR es de conceptualizar las conductas distintas entre machos y hembras humanas como "significativos" (como es significativo el canto de los pinzones cebra) y entonces explicar dichos datos ampliándolos conceptualmente y subsumiéndolos bajo el patrón nómico de la OR. Ahora bien, lo que se intenta señalar como ideología de género es, justamente, que los y las miembros del programa endocrino traten los datos sobre seres humanos del mismo modo en el que se tratan los datos provenientes de otras especies de mamíferos. Es decir, de nuevo, deberían darse argumentos independientes para ello. Y no los hay.

Nuestra principal crítica al abordaje es que del hecho bien asumido de que el mismo conjunto de fenómenos sea explicable por distintas teorías (infra determinación de la teoría por los hechos) no demuestra que las razones de proponer una teoría u otra sean ideológicas. En el caso particular de Longino además, hay varios elementos que desalientan esta lectura. Por un lado, elegir una teoría más general, con mayor poder de sistemati- 
zación que otra, parece una decisión guiada por valores epistémicos, no contextuales. Por otro, en el contexto particular de la biología, el "presupuesto de extrapolación" entre otros animales y los seres humanos difícilmente puede considerarse algo más que hacer ciencia después de la revolución darwinista. Finalmente, las teorías propuestas como alternativas no son incompatibles, la poda neural a partir de la etiquetación o la medicalización de la infantil no presenta, en principio, contradicción con la acción organizacional de las hormonas en fases críticas del período embrionario.

Con todo, el problema del neurosexismo y de los tipos de cerebros subsiste. ¿Cómo precisar dónde se agazapan los valores patriarcales en la neuroendocrinología?

\section{Una reconstrucción alternativa}

A continuación, presentaremos una reconstrucción alternativa a la ofrecida por Longino, pues creemos que utilizando otro andamiaje metateórico, podemos alcanzar algún grado de claridad respecto al problema discutido. Para ello recurriremos al estructuralismo metateórico, aunque en una versión eminentemente abreviada.

Para el estructuralismo, la noción mínima de teoría se corresponde con el par ordenado $\langle K, I\rangle$. $K$ representa el aparato conceptual, legal y matemático de la teoría, mientras que $I$ representa aquellos sistemas del mundo a los que la teoría pretende aplicarse. Aquí se presentará solo parcialmente $K$ (los modelos y los modelos potenciales) e $I$. Comenzaremos por un ejemplar paradigmático y luego presentaremos una reconstrucción informal y una formal ${ }^{2}$.

\subsection{Un ejemplar paradigmático}

Uno de los casos aplicativos que recorre las revisiones críticas e ilustra los manuales es la conducta reproductiva de las ratas, como podemos ver a continuación:

\footnotetext{
${ }^{2}$ Para una presentación concisa del enfoque, véase Díez y Lorenzano (2002). Para la presentación más completa y acabada, véase Balzer et al. (1987).
} 


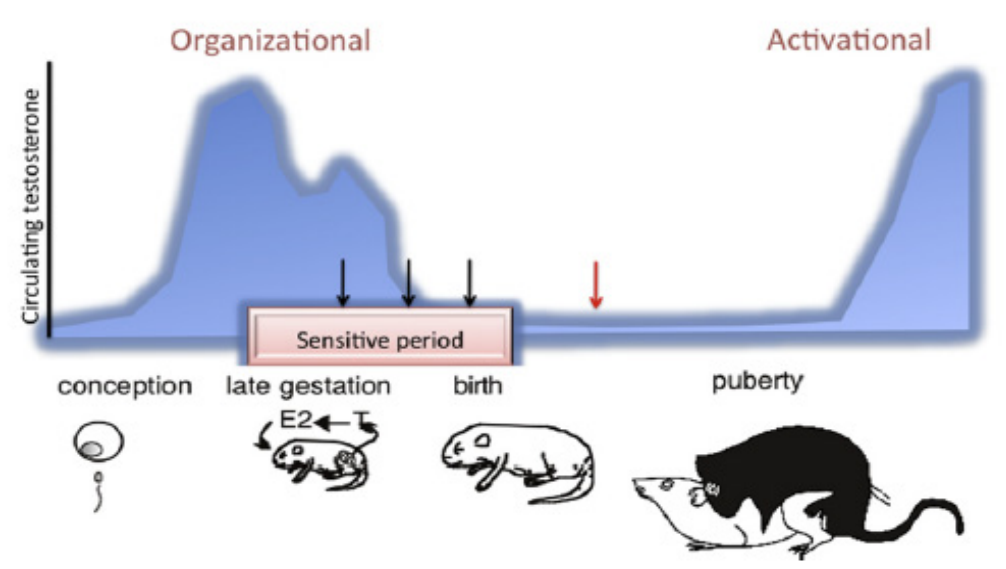

Fig. 3. Representación gráfica de la acción organizacional y activacional de las hormonas gonadales atendiendo al momento en el que se produce y las conductas resultantes en las wistar macho. (Extraído de Fink et al. 2011, 395)

El caso de las wistar macho tiene todos los componentes de ejemplar paradigmático de Kuhn. En primer lugar, ocupa un rol destacado entre los manuales con los cuales se forman las nuevas generaciones de científicos, constituyéndose en la referencia básica en el plano pedagógico. En segundo lugar, el ejemplo condensa todos los conceptos de la teoría, así como sus afirmaciones acerca del comportamiento de los sistemas de referencia, es decir, es un buen ejemplo de la teoría. Finalmente, y debido a ambas consideraciones previas, el ejemplar de la wistar macho tiene un rol heurístico fundamental, en tanto guía las nuevas aplicaciones a partir de las indicaciones que genera: si usted tiene una conducta dimórfica, busque la acción hormonal sobre el cerebro en distintos momentos del desarrollo.

Tomemos el artículo de Houtsmuller et. al (1994). En dicho trabajo se encuentra una aplicación del caso de las wistar macho:

Los machos fueron tratados prenatal o prenatal y neonatalmente con ATD. Los patrones de conducta sexual y elección de parejas fueron estudiados en la adultez. Luego, los animales fueron sacrificados y el volumen del SDN-POA fue medido. El volumen del SDN-POA resultó reducido en ambos grupos. La combinación de tratamiento pre y neonatal dio lugar a una frecuencia reducida de monta, intromisión y eyaculación, así como redujo la preferencia de hembras sobre machos. El tamaño del SDN-POA se correlaciona significativa y positivamente con la frecuencia de la conducta sexual masculina, así como con la preferencia por las hembras frente a los machos. (Houtsmuller et al. 1994, 535. La traducción es nuestra)

Nótese los componentes: conductas dimórficas (monta, intromisión, eyaculación, preferencia de hembras o machos), estructura neurofisiológica dimórfica (SDN-POA, núcleo 
sexualmente dimórfico del área preóptica por sus siglas en inglés) y hormonas (en este caso, ATD es un bloqueador de la aromatización de la testosterona a estradiol). Cuando un bloqueador de la aromatización de la testosterona es administrado a las wistar preñadas y a las crías macho recién nacidas, se obtienen machos con un descenso o desaparición de monta y eyaculación respecto del grupo control. Post morten, se observa que el SDNPOA de las wistar tratadas con el bloqueador tiene un volumen menor que el de las wistar control. Formulado de otra forma: La acción organizacional de la testosterona durante el período pre y perinatal, es responsable de la masculinización del SDN-POA, el cual es el responsable de las conductas de monta y eyaculación.

\subsection{Reconstrucción Informal}

\subsubsection{Aplicaciones pretendidas (explanandum)}

La OR es una teoría que pretende explicar cómo se originan y como se expresan las conductas sexualmente dimórficas $(B)$. $B$ es el conjunto todas las conductas que son dimórficas atendiendo al sexo, incluyendo en la noción de dimorfismo aquellas que son diferencias absolutas (un sexo tiene la conducta y el otro no), de forma (aquellas conductas que ambos sexos manifiestan, pero de forma distinta) o de frecuencia (aquellas conductas que ambos sexos manifiestan de forma idéntica pero en diferente cantidad). Como se ve, B presupone la existencia de dos sexos (y sólo dos) y todos los miembros del conjunto, es decir, toda conducta dimórfica, tiene tres valores posibles: si b pertenece a $B$, entonces $b$ puede ser $b_{i}$ (indiferenciada o prediferenciada), $b_{m}$ (masculina), $b_{f}$ (femenina).

\subsubsection{Núcleo conceptual y legal de la teoría (explanans)}

La OR sostiene que las conductas sexualmente dimórficas dependen de la interacción (INT) de las hormonas gonadales $(H)$ con el cerebro $(C)$ de un individuo $(I)$ durante los períodos relevantes de tiempo $(T)$. $\mathrm{H}$ es el conjunto de las hormonas gonadales, independientemente de si son estas son andrógenos o estrógenos. Notablemente, la OR no otorga relevancia a las distinciones usuales entre hormonas gonadales masculinas y hormonas gonadales femeninas en tanto unas pueden, eventualmente, transformarse en otras y la masculinización o feminización de las estructuras neurofisiológicas dimórficas no depende de qué hormona u hormonas actúen o de donde estas procedan, sino más bien de que $H$ y $C$ participen de la relación $I N T$ en un tiempo dado o no lo hagan (Principio de Adán).

Los períodos de tiempo que la teoría reconoce son tres $t_{1}, t_{2}, t_{3}$, que respectivamente están por indiferenciado, organizacional y activacional, suponiendo que los períodos son consecutivos en ese orden. Aquí deben señalarse que $t_{1}$ y $t_{2}$, es decir, el período organizacional, son fijos para cada especie y variables a través de las especies, mientras que $t_{2}$ es variable para cada y a través de las especies. Si bien esto no es explicitado en ningún pasaje de la literatura relevante, es evidente que los períodos organizacional y activacional 
no son conceptualmente simétricos: el auge hormonal que activa las conductas dimórficas puede ocurrir a lo largo de toda la vida post natal mientras que el auge hormonal que organiza las estructuras neurofisiológicas dimórficas solo puede darse en un momento específico del desarrollo, atendiendo a cada especie. Además, mientras que la ausencia de hormonas en el período organizacional conduce a la diferenciación masculina, la ausencia de hormonas en el período activacional implica la no manifestación de la conducta independientemente del sexo.

Las estructuras neurofisiológicas sexualmente dimórficas $(S)$ son el conjunto de aquellas áreas cerebrales que difieren entre machos y hembras y que son responsables de las conductas dimórficas. Al igual que en el caso de $B$, presuponen la existencia de dos y sólo dos sexos, y, si $s$ pertenece a $S$, entonces puede tener tres valores $s_{i}$ (indeferenciada), $s_{f}$ (femenino), $s_{m}$ (masculino). Como resultado de la investigación sistemática bajo la OR, se ha generado una profusa taxonomía acerca de qué estructuras son dimórficas y en qué sentido son dimórficas. Toda esta pluralidad, no obstante, no genera disparidades conceptuales relevantes: en el marco de la teoría, todas las distintas estructuras en todas las formas en que son dimórficas caben en $S$. Finalmente, la OR propone los conceptos de organización $O R G$ y activación $A C T$ para reflejar el modo en el cual las hormonas producen la diferenciación de las estructuras neurofisiológicas y las activan, dependiendo del período de tiempo en el que la interacción hormonal ocurra.

De modo informal, la ley fundamental de la OR se presenta de la siguiente manera:

1. En el periodo pre sexual, no hay ni estructuras ni conductas dimórficas.

2. Para toda estructura dimórfica y para todo individuo, existen cerebros y hormonas tales que,

2.1 se organiza una estructura neurofisiológica masculina si el cerebro interacciona con las hormonas en $t_{1}$;

2.2 si no interaccionan, entonces se organiza una estructura neurofisiológica femenina;

2.3 las estructuras que el cerebro adquiere en $t_{1}$ son resultado de la organización;

2.4 la conducta exhibida en $t_{2}$ por un individuo es el resultado de la activación o de la organización del cerebro de este. 


\subsection{Reconstrucción formal}

\subsubsection{Modelos potenciales}

Los modelos potenciales son el tipo de estructura del que tiene sentido preguntarse si cumplen o no con las leyes de la teoría. Es decir, los $M_{p}$ representan el aparato conceptualizador de la teoría. Los $M_{p}$ se componen de los dominios de objetos (conjuntos) y de las relaciones y funciones:

\section{Dominios de objetos}

- $B$ : es el conjunto tipos de conductas que pueden tener tres valores: indiferenciada, masculina y femenina.

- I es el conjunto de individuos de la misma especie, asumiendo que dicha especie está sexualmente dividida.

- $C$ es el conjunto de los cerebros.

- $S$ es un conjunto de estructuras neurofisiológicas que pueden tener tres valores: indiferenciada, masculina y femenina.

- $H$ es el conjunto de hormonas gonadales.

- $\quad T$ y > están por los tres períodos que la teoría reconoce, prediferenciado, organizacional y activacional y su sucesión temporal (se asume que $t_{0}, t_{1}, t_{2}$ se suceden en el tiempo de forma ordenada)

\section{Relaciones y funciones}

- INT es una relación de cerebro y hormonas en un tiempo.

- $C E R$ es función y asigna cerebros a individuos.

- $\quad S T R$ es función y asigna estructuras a cerebros en un cierto tiempo.

- $C O D$ es función y asigna conductas a individuos cierto tiempo.

- $O R G$ es función y asigna estructuras a cerebros.

- $A C T$ es función y asigna conductas a un cerebro.

Con estos elementos podemos brindar la primera definición de la reconstrucción: 


\section{Definición 1}

$M_{p}(\mathrm{OR}): x$ es un modelo potencial de la teoría organizacional $\left(x \in M_{p}(\mathrm{OR})\right)$ sí y solo sí existen $B, I, C, S, H, T,>, I N T, C E R, S T R, C O D, O R G, A C T$ tal que

(0) $x=\langle B, I, C, S, H, T,>, I N T, C E R, S T R U C T, C O D, O R G, A C T\rangle$

(1) $B$ es un conjunto de cardinalidad $3\left(B=\left\{b_{i}, b_{f}, b_{m}\right\}\right)$

(2) $I$ es un conjunto finito no vacío

(3) $C$ es un conjunto finito, no vacío

(4) $S$ es un conjunto de cardinalidad $3\left(S=\left\{s_{i}, s_{m}, S_{f}\right\}\right)$

(5) $H$ es un conjunto finito y no vacío

(6) $T$ es un conjunto de cardinalidad $3\left(T=\left\{t_{0}, t_{1}, t_{2}\right\}\right)$

(7) $>\subseteq t_{2}$

(8) $I N T \subseteq C \times T \times P o t(H)$

(9) $C E R: I \rightarrow C$

(10) STR: $C \times T \rightarrow S$

(11) $C O D: I \times T \rightarrow B$

(12) $O R G: C \rightarrow S$

(13) $A C T: C \rightarrow B$

\subsubsection{Los modelos actuales}

Habiendo caracterizado los dominios de objetos, relaciones y funciones de la teoría, al introducir el predicado conjuntista que define los modelos potenciales, podemos preguntarnos ahora cuáles de las estructuras que cumplen con estos axiomas impropios, cumplen además con los axiomas propios, es decir, con las leyes de la teoría.

\section{Definición 2}

$M(\mathrm{OR}): x$ es un modelo de la teoría organizacional $(x \in M(\mathrm{OR}))$ syss existen $B, I, C$, $S, H, T,>, I N T, C E R, S T R, C O D, O R G, A C T$, tal que

(0) $x \in M_{p}(\mathrm{OR})$

(1) $\forall c \in C, \operatorname{STR}\left(c, t_{0}\right)=s_{i} \wedge \forall i \in I, C O D\left(i, t_{0}\right)=b_{i}$

(2) $\forall b_{k} \in B, \forall i \in I, \exists c \in C, \exists h \in H$, tal que: 
(2.1)

(2.2) $\left\langle c, t_{l}, h\right\rangle \notin I N T \rightarrow O R G(c)=s_{f}$

(2.3) $\operatorname{STR}\left(c, t_{1}\right)=O R G(c)$

(2.4) $C E R(i)=c \wedge C O D\left(i, t_{2}\right)=A C T(c) \vee O R G(c)$

\section{Cerebros tipo o cerebros mosaico}

Ya discutimos cómo la asignación de un cerebro masculino o femenino a partir de la bifurcación hormonal es uno de los argumentos centrales contra la OR. Hasta ahora, lo hemos discutido en el marco de la metaciencia, pero el problema de los cerebros-tipo es también un problema científico que puede plantearse como la siguiente pregunta ¿Cómo se deben conceptualizar las diferencias cerebrales entre hombres y mujeres? Además, la discusión ha traspasado las fronteras de lo académico en general para llegar a instalarse en los medios de comunicación masiva.

Sin dudas, el artículo que más ha agitado el avispero del debate es "Sex beyond the genitalia: The human brain mosaic" (151 veces citado al momento de escribir este párrafo) de Daphma Joel et al. y su equipo de Tel Aviv. En torno a este y a las respuestas al mismo, desarrollaremos nuestro análisis. Bien, los términos de la polémica son los siguientes: habida cuenta del hallazgo de diferencias neuroanatómicas relacionadas con el sexo a partir de la aplicación sistemática de la OR y del estudio en general del cerebro humano ¿estamos legitimados a conceptualizar los cerebros de hombres y mujeres como perteneciendo a tipos distintos? La respuesta afirmativa a esa pregunta puede encontrarse en autores como Glezerman (2016), Arnold (2009), Cahill (2006), Baron Cohen (2005). Todos ellos asumen que la existencia de estructuras neurofisiológicas dimórficas legitima la distinción entre cerebro-tipo-femenino y cerebro-tipo-masculino. Por el lado de la respuesta negativa, las representantes más destacadas son Joel $(2011 ; 2012)$, Joel et al. (2015) y Anne Fausto-Sterlling (1992).

Sobre un total de 1400 cerebros, Joel y su equipo realizan diversas mediciones (volumen total del cerebro, distribución de materia gris, comparación de las estructuras más claramente dimórficas etc.). Frente a la visión clásica (OR) pretenden mostrar que el cerebro no puede ser catalogado como dimórfico. Para ello, debe mostrar que hay escasa consistencia interna entre las características típicamente masculinas y las características típicamente femeninas. Además, debe mostrar que existe un fuerte solapamiento entre dichas características, esto es, que hay más cerebros con características de ambos tipos. Las conclusiones son sólidas:

Nuestro estudio demuestra que aunque existen diferencias de sexo / género en la estructura cerebral, los cerebros no se dividen en dos clases, una típica de los hombres y la otra típica de las mujeres, ni están alineadas a lo largo de un continuo "cerebro masculino-cerebro 
femenino". Por el contrario, incluso considerando solo el pequeño grupo de características cerebrales que muestran las mayores diferencias sexo / género, cada cerebro es un mosaico único de características, algunas de las cuales pueden ser más comunes en las mujeres en comparación con los hombres, otras pueden ser más comunes en los hombres en comparación con las mujeres, y aún otros pueden ser comunes tanto en mujeres como en hombres. (Joel et al. 2015, 5. La traducción es nuestra)

Nótese que en ningún momento se niega que existan diferencias sexuales en el cerebro, lo que se pretende mostrar es que tales diferencias muestran una fuerte variabilidad y pobre consistencia interna, haciendo más razonable conceptualizar los cerebros como mosaicos individuales que como tipos dimórficos como las gónadas. Lo que los y las autoras muestran es que, en promedio, tomando un cerebro cualquiera y analizándolo exhaustivamente, no podríamos determinar a qué sexo pertenece.

El trabajo de Joel ha generado, además, grandes repercusiones. Se han presentado objeciones sustantivas a la metodología para distinguir dimorfismos (Del Giudice et al. 2016) como al enfoque morfológico de la propuesta mosaico de Joel (Glezerman 2016). La discusión ha seguido con sucesivas respuestas por parte de Joel et al. (2016a, 2016b) y con nuevas revisiones críticas de la metodología original (Rosenblatt 2016; Chekroud et al. 2016). Aquí, el debate metodológico resulta de escaso interés para el tema que nos ocupa. Lo que nos interesa aquí es si efectivamente, y como Joel y las críticas de la OR sostienen, nuestra teoría objeto favorece la visión tipológica de los cerebros.

Retomemos nuestra reconstrucción de la OR. Allí establecimos que los $M_{p}$ establecen la clase de estructuras de las que cabe preguntarse si cumplen además las constricciones nómicas de la teoría. En el caso de la OR establecimos que tales estructuras son del siguiente tipo: $\langle B, I, C, S, H, T\rangle,, C E R, S T R, C O D, O R G, A C T\rangle$ y caracterizamos cada uno de los dominios que la componen y las relaciones y funciones sobre tales dominios

Lo primero que resulta obvio, si nuestra reconstrucción es correcta, es que la parte conceptualizadora-tipificadora de la teoría no incluye ninguna referencia a cerebros-tipo. Es decir, la teoría no exige que los individuos a las cuales se va a aplicar posean cerebros tipo femenino o cerebros tipo masculino. Se podría aducir que esta afirmación es tramposa, en tanto las $\mathrm{S}$ pueden tomar tres valores: masculino, femenino o indiferenciado y la función ORG asigna tales estructuras a los cerebros de los individuos. Es decir, se podría afirmar que la noción de cerebro-tipo está conceptualizada de tal manera que evita el término, pero permanece conceptualmente: un cerebro al que la organización ha asignado estructuras de tipo masculino sería un cerebro-tipo-masculino. Ahora bien, recordemos que la polémica no es en torno a la existencia de diferencias relativas al sexo en el cerebro, sino más bien a si tales diferencias favorecen la conceptualización de los cerebros como perteneciendo a tipologías distintas o más bien como mosaicos variables de estructuras dimórficas. 
Para contestar prolijamente a esta cuestión debemos referirnos ahora a la ley de la OR. Lo que la ley establece que (1) En el periodo pre sexual, no hay ni estructuras ni conductas dimórficas y (2) Para toda estructura dimórfica y para todo individuo, existen cerebros y hormonas tales que (2.1) Se organiza una estructura neurofisiológica masculina si el cerebro interacciona con las hormonas en $\mathrm{t}_{1},(2.2)$ si no interaccionan, entonces se organiza una estructura neurofisiológica femenina. (2.3) las estructuras que el cerebro adquiere en $t_{1}$ son resultado de la organización (2.4) la conducta exhibida en $t_{2}$ por un individuo es el resultado de la activación o de la organización del cerebro de este. Es decir, si hay interacción hormonal en el período organizacional, entonces se organiza una estructura de tipo masculino y si no la hay, femenino. Aquí no se establece que las conductas dimórficas se expliquen por un determinado tipo de cerebro. Más bien se establece el modo por el cual se diferencian ciertas estructuras que entonces resultan explicativas de tales conductas.

Siendo así ¿cuál es nuestro punto? El estructuralismo propone una noción clara de identidad de una teoría: la entidad más simple que puede ser catalogada como tal es el par ordenado $\langle K, I\rangle$ donde $K$ es el aparato conceptual-legal de la teoría e $I$ el conjunto de los sistemas del mundo a los que la teoría pretende aplicarse. En general, además, las teorías son redes jerárquicas de elementos teóricos de este tipo. Justamente por su ser jerárquico, todo lo que la teoría dice está contenido en el elemento teórico básico. Así pues, para determinar si una teoría efectivamente dice algo debemos analizar los elementos contenidos en la dupla $\langle K, I\rangle$. Si nuestra interpretación de la teoría es correcta, el concepto de cerebros-tipos no forma parte de lo que la teoría pretende explicar, es decir, no está incluido en las las aplicaciones pretendidas, entendidas como $I$. Por otro lado, tal concepto es ajeno al aparato legal de la teoría: nada hay en la ley que sea equiparable a la introducción de cerebros-tipo. Al menos desde el punto de vista analítico, la OR es ajena a la noción discutida.

Ahora bien, lo que acabamos de decir no es suficiente para despejar todas las dudas. Es cierto que la OR no habla sobre tipos de cerebros, pero eso no satisface las dudas al respecto. Al fin y al cabo, aun cuando la OR pueda sostenerse independientemente de que se muestre poco razonable la distinción tajante de tipos de cerebros, en algún sentido parece que el dimorfismo cerebral es una consecuencia de la OR: si aplicamos la teoría de forma iterada sobre distintos individuos de una dada especie, entonces obtendríamos una imagen global de las diferencias neurofisiológica de dicha especie, lo que nos permitiría determinar cómo es el cerebro de los machos y cómo el de las hembras de la especie. Aquí lo que se pone en juego es si el programa de investigación bajo la OR conduce a tal visión dicotómica del cerebro.

Esta es justamente la postura de Glezerman, Baron-Cohen y Cahill entre otros. La teoría, en su uso sistemático y repetido, legitima hablar de cerebros femeninos y masculinos. Ahora bien, lo que queremos mostrar no es que tal lectura de las consecuencias del programa neuroendocrino sea errada, sino más bien que no son las únicas posibles. De hecho, la OR trata a las conductas dimórficas y a las estructuras neurofisiológicas dimórficas

Revista de Humanidades de Valparaíso, 2019, No 14, 287-313 
que las explican como independientes de otras, lo cual se evidencia en algunos ejemplos citados: el timing y la cantidad de testosterona administrada a una conejilla de indias preñada, produce efectos distintos en la descendencia respecto a las conductas dimórficas, pudiendo coexistir (como de hecho ocurre, incluso en el taller de la naturaleza) conductas dimórficas de valor distinto en el mismo individuo. Estos ejemplos podrían favorecer entonces más bien una visión mosaica de las consecuencias de la OR. De hecho, algunas de las más reputadas figuras de la disciplina han tomado este camino, por ejemplo, Margaret McCarthy (Joel et al. 2017)

Nuestro análisis nos lleva a dos cuestiones ulteriores, muy interesantes para el problema discutido. Por un lado, nuestra elucidación nos permite señalar de modo más preciso dónde están los valores no epistémicos, comparado con el análisis informal y deficitario de Longino: no es que la OR presuponga los viejos cánones ideológicos del sexismo, sino más bien que la interpretación que hagamos de sus consecuencias qua programa de investigación puede estar guiada o no por tales valores u otros. El hecho de que algunos científicos (Brizendine 2007) y divulgadores profesionales tomen el camino de la tipología frente al mosaiquismo, parece indicar algo relevante acerca de ciertas presuposiciones no puramente teóricas. A partir de la interpretación dicotómica del cerebro, defienden la naturalidad del statu quo opresivo hacia las mujeres y otras lindezas, como la educación segregada (Sax 2006). Al margen de cómo la elucidación de la OR nos permite determinar que la polémica de los cerebros tipo no afecta a dicha teoría, no quiere decir que la polémica no sea interesante o relevante, ni que sea indigna de análisis filosófico. Es interesante que Joel misma señale que su preocupación sobre la legitimidad de la categorización surge de la obvia relevancia social que tiene la posibilidad de que exista un sexo cerebral y que ello sea utilizado como baza científica en favor de, por ejemplo, la educación segregada por sexos:

En el plano social, adoptar una visión que reconozca la variabilidad y la diversidad humanas tiene implicaciones importantes para los debates sociales sobre cuestiones de larga data, como la conveniencia de la educación de un solo sexo y el significado de sexo / género como categoría social. (Joel et al. 2015, 15472. La traducción es nuestra)

Pese a que consideramos que Joel erra al caracterizar la conceptualización de los cerebros-tipo como un resultado natural de la OR, coincidimos en que la discusión es relevante y que tiene una obvia dimensión social. De hecho, consideramos que hemos mostrado que tal debate es preminentemente ideológico: el programa neuroendocinológico del comportamiento puede subsistir sin pronunciarse al respecto. Sin embargo, sería útil aquí adelantar alguna de las conclusiones del presente capítulo. Nótese que Joel subordina el significado de su trabajo a mostrar que no hay tal cosa como cerebro femenino y cerebro masculino, porque ello desterraría para siempre de la neurociencia las posiciones ideológicamente deleznables de Brizendine, Sax y otros. A esta afirmación subyace una idea como mínimo peligrosa: que las decisiones acerca de cosas como la educación mixta, el 
desarrollo de políticas públicas orientadas a la erradicación de la desigualdad histórica sufrida por parte de la mujer, a la discriminación positiva etc. dependen de las teorías vigentes en una dada (o en varias) disciplinas. De hecho, es la misma posición de Sax, pero en sentido contrario: "si tenemos una teoría que avala que las mujeres y los hombres tienen cerebros distintos, entonces la educación segregada es deseable" frente a "si tenemos una teoría que avala que el cerebro humano es un mosaico, entonces la educación mixta es deseable". Ambas posturas son erradas, aun cuando una encarne valores progresistas con los que nos sentimos mucho más identificados.

\section{Conclusiones}

Al tomarnos en serio la propuesta del ECC de Longino, hemos descubierto que los análisis informales o superficiales no son suficientes para determinar dónde se agazapan esos valores que deben ser explicitados para poderlos discutir racional e intersubjetivamente. La reconstrucción propuesta como alternativa a la de Longino, nos permite evitar el error de calificar de valores patriarcales a lo que son valores netamente epistémicos (como la generalidad) y nos posiciona mejor para señalar dónde están los valores entreverados en la práctica de la neuroendocrinología: no es en la teoría misma, sino en el modo en el que se presenta y en el modo en el que se utiliza como ariete ideológico para legitimar posiciones del orden conservador.

Es por ello por lo que consideramos importante discutir y clarificar la noción de neurosexismo. Lo que hemos mostrado es que al menos en el caso presentado (no necesariamente en todos los que se puedan presentar) el sentido de neurosexismo que ocurre es el del género de divulgación o de ciencia Lega protagonizado por Brizendine, Sax y otros. Eso nos permite mantenernos en una posición feminista de repudio al neurosexismo sin rechazar por ello el estudio de las diferencias y la diferenciación sexual.

Esta revisión nos permite además navegar la tensión entre los dos sentidos de androcentrismo que presentamos al principio. Al no rechazar de plano los estudios de las diferencias sexuales, sino más bien su utilización como excusa para defender el patriarcado, estamos en mejores condiciones para defender el desarrollo de la medicina que no ponga al hombre en el centro y que reconozca las especificidades de las mujeres y las disidencias. No sólo las socioambientales, sino también las referidas a la constitución biológica.

Nuestra postura podría entenderse como un modo de salvar los muebles frente a los valores no cognitivos, sacándolos de las teorías y volviendo a una versión aggiornada de la ciencia libre de valores. Tal lectura nos parece errada, pues cuando decimos que los valores no están en la teoría, no queremos decir que en la neuroendocrinología como disciplina no haya valores en juego, sino que no están allí donde se los busca. Las reconstrucciones racionales no pueden reflejar la complejidad y riqueza de la práctica científica, sino cierto aspecto de esta. Que no esté en la teoría, en nuestro caso, significa que los 
conceptos que se consideran típicamente androcéntricos (como tipos de cerebro) no son conceptos propuestos por la teoría entendida como el par ordenado $\langle K, I\rangle$. Eso no quiere decir que la práctica científica no ponga en juego valores. De hecho, hemos propuesto dónde están y cómo atacarlos siguiendo a algunas autoras feministas.

\section{Referencias bibliográficas}

Arnold, A. P. (2009). The organizational-activational hypothesis as the foundation for a unified theory of sexual differentiation of all mammalian tissues. Hormones and Behavior, 55(5): 570-578. doi: 10.1016/j.yhbeh.2009.03.011

Balzer, W., Moulines, C. U., Sneed, J. (1987). An architectonic for science. The structuralist program. Dordrecht: Reidel.

Baron-Cohen S., Knickmeyer R.C., Belmonte M.K. (2005). Sex differences in the brain: implications for explaining autism. Science, 310: 819-823.

Bernabé, F., Giri, L. (2019). It's a Male World: el sesgo sexual de los modelos animales en biología. Arbor, 195(791): a492. doi: https://doi.org/10.3989/arbor.2019.791n1005

Borgerson, K. (2011). Amending and defending Critical Contextual Empiricism. European Journal of Philosophy of Science, 1(3):435-449.

Brizendine, L. (2007). The female brain. London: Bantam Press.

Brookey, R. A. (2002). Reinventing the Male Homosexual: The Rhetoric and Power of the Gay Gene. Bloomington: Indiana University Press.

Cahill, L. (2006). Why sex matters for neuroscience. Nature Review Neurosciesce, 7(6): 477-484. doi: 10.1038/nrn1909

Chekroud, A. M., Ward, E. J., Rosenberg, M. D., Holmes, A. J. (2016). Patterns in the human brain mosaic discriminate males from females. PNAS, 113(14): E1968. doi: https://doi. org/10.1073/pnas. 1523888113

Darwin, C. (2009). El origen del hombre. Madrid: Crítica.

Del Giudice, M., Lippa, R. A., Puts, D. A., Bailey, D. H., BAiley, J. M., Schmitt, D. P. (2016). Joel et al.'s method systematically fails to detect large, consistent sex differences. PNAS, 113(14): E1965. doi: https://doi.org/10.1073/pnas.1525534113

Diez, J. A., Lorenzano, P. (eds.) (2002). Desarrollos actuales de la metateoría estructuralista: Problemas y discusiones. Bernal: Universitat Rovira i Virgili y Universidad Nacional de Quilmes.

Fausto-Sterling, A. (1992). Myths of Gender. Nueva York: BasicBooks.

Fine, C. (2008). Will working mothers' brains explode? The popular new genre of neurosexism. Neuroethics, 1(1): 69-72. 
Fine, C. (2010). Delusions of Gender: How Our Minds, Society, and Neurosexism Create Difference. New York: W. W. Norton.

Fink, G., Pfaf, D., Levine, J. (2011). Handbook of Neuroendocrinology. Oxford: Academic Press.

Glezerman, M. (2016). Yes, there is a female and a male brain: Morphology versus functionality. PNAS, 113(14): E1971. doi: https://doi.org/10.1073/pnas.1524418113

Gómez, R. (2015). La dimension valorativa de las ciencias. Bernal: Universidad Nacional de Quilmes.

Hankinson Nelson, L. (1990). Who Knows: From Quine to Feminist Empiricism, Philadelphia:Temple University Press.

Hankinson Nelson, L. (1993). Epistemological communities. En Linda Alcoff and Elizabeth Potter (eds), Feminist Epistemologies, pp. 121-59. New York and London: Routledge.

Harding S. (1986). The Science Question in Feminism. Ithaca: Cornell University Press.

Harding, S. (1993). Rethinking standpoint epistemology: what is 'strong objectivity'? En Linda Alcoff y Elizabeth Potter (eds.), Feminist Epistemologies, pp. 49-82. New York and London: Routledge.

Hess, B. B. (1990). Beyond dichotomy: Drawing distinctions and embracing differences. Sociological Forum, 5(1): 75-93. http://www.jstor.org/stable/684582.

Houtsmuller E. J., Brand, T., de Jonge F. H., Joosten, R. N., van de Poll, N. E., Slob, A. K. (1994). SDN-POA Volume, Sexual Behavior, and Partner Preference of Male Rats Affected by Perinatal Treatment with ATD. Physiology \& Behavior, 56 (3): 535-541.

Joel, D. (2011). Male OR female? Brains are intersex. Frontiers in Integrative Neuroscience, 5: 57. doi: https://doi.org/10.3389/fnint.2011.00057

Joel, D. (2012). Genetic-gonadal-genitals sex (3G-sex) and the misconception of brain and gender, or, why 3G-males and 3G-females have intersex brain and intersex gender. Biology of Sex Differences, 3: 27.

Joel, D., Berman, Z., Tavor, I., Wexler, N., Gaber, O., Stein, Y., Shefi, N., Pool, J., Urchs, S., Margulies, D. S., Liem, F., Hänggi, J., Jäncke, L., Assaf, Y. (2015). Sex beyond the genitalia: The human brain mosaic. PNAS, 112(50): 15468-15473. doi: https://doi.org/10.1073/ pnas. 1509654112

Joel, D., Hänggi, J., Pool, J. (2016). Reply to Glezerman: Why differences between brains of females and brains of males do not 'add up' to create two types of brains. Proc Natl Acad Sci USA (Letter).

Joel, D., Persico, A., Hänggi, J., Pool, J., Berman, Z. (2016) Reply to Del Guidice et al., Chekroud et al., and Rosenblatt: Do brains of females and males belong to two distinct populations? Proc Natl Acad Sci USA (Letter). 
Joel, D., McCarthy, M. M. (2017). Incorporating Sex as a Biological Variable in Neuropsychiatric Research: Where Are We Now and Where Should We Be? Neuropsychopharmacology, 42(2): 379-385.

Jordan-Young, R. (2010). Brain Storm. Cambridge, Massachusetts: Harvard University Press.

Klinge, I., Wiesemann, C. (2010). Sex and Gender in Biomedicine. Göttingen: Universitätverlag Göttingen.

Legato, M. (2004). Principles of Gender-Specific Medicine. San Diego: Elsevier Academic Press.

Longino, H. (1994). In Search of Feminist Epistemology. The Monist, 77(4): 472-485.

Longino, H. E. (1997). Feminismo y filosofía de la ciencia. En Marta González García et. al (eds.), Ciencia, tecnología y sociedad, 71-83. Barcelona: Ariel.

Potter, E. (2006). Femminism and Philosophy of Science. Routledge: New York.

Regitz-Zagrosek, V. (2012). Sex and gender differences in health. EMBO Reports, 13(7): 596603. doi: 10.1038/embor.2012.87

Reinharz, S. (1986). Patriarcal Pontifications. Society, 23(6): 23-29. doi: https://doi.org/10.1007/ BF02697107

Rosenblatt, J. D. (2016). Multivariate revisit to "sex beyond the genitalia". Proc. Natl. Acad. Sci. USA, 113(14): E1966-E1967. doi: 10.1073/pnas.1523961113

Sax, L. (2006). Why gender matters: What parents and teachers need to know about the emerging science of sex differences. New York: Broadway Books.

Tacsir, E., Grazzi, M., Castillo, R. (2014). Women in Science and Technology: What Does the Literature Say? Inter-American Development Bank, Technical Note No. IDB-TN-637. https://publications.iadb.org/publications/english/document/Women-in-Science-andTechnology-What-Does-the-Literature-Say.pdf

van der Wijngaard, M. (1997). Reinventing the Sexes: The Biomedical Construction of Femininity and Masculinity. Bloomington: Indiana University Press.

Wizemann T. M., Pardue, M. (2001). Exploring the Biological Contributions to Human Health. Does Sex Matter? Washington: National Academies Press.

Wylie, A. (1997). The engendering of archaeology: refiguring feminist science studies. Osiris, 12: 80-99. http://www.jstor.org/stable/301900

Wylie, A. (2003). Why standpoint matters. En Robert Figueroa and Sandra Harding (eds.), Science and Other Cultures: Issues in Philosophies of Science and Technology, pp. 26-48. New York: Routledge. 